\title{
Philosophiques
}

Jean-François PRADEAU (dir.), Platon : les formes intelligibles. Sur la forme intelligible et la participation dans les dialogues platoniciens, Paris, Presses Universitaires de France, collection Débats philosophiques, 2001, 192 pages.

\section{Georges Leroux}

Volume 31, numéro 1, printemps 2004

Poincaré et la théorie de la connaissance

URI : https://id.erudit.org/iderudit/008946ar

DOI : https://doi.org/10.7202/008946ar

Aller au sommaire du numéro

Éditeur(s)

Société de philosophie du Québec

ISSN

0316-2923 (imprimé)

1492-1391 (numérique)

Découvrir la revue

Citer ce compte rendu

Leroux, G. (2004). Compte rendu de [Jean-François PRADEAU (dir.), Platon : les formes intelligibles. Sur la forme intelligible et la participation dans les dialogues platoniciens, Paris, Presses Universitaires de France, collection Débats philosophiques, 2001, 192 pages.] Philosophiques, 31(1), 251-254. https://doi.org/10.7202/008946ar d'utilisation que vous pouvez consulter en ligne. 
Jean-François PRADEAU (dir.), Platon: les formes intelligibles. Sur la forme intelligible et la participation dans les dialogues platoniciens, Paris, Presses Universitaires de France, collection Débats philosophiques, 2001, 192 pages.

Le format particulier de cette collection propose des recueils de contributions brèves, introductives sans être élémentaires, et pleinement représentatives de la recherche contemporaine sur un auteur ou une question controversée. L'interprétation de la métaphysique platonicienne appartient à ce lot de grandes quaestiones vexatae, dans lesquelles chaque époque développe une herméneutique et élabore une critique. La doctrine des formes intelligibles constitue en effet le morceau le plus essentiel de cette métaphysique, et, malgré que des siècles aient été consacrés à l'interpréter, elle ne cesse d'être reprise. Coordonné par Jean-François Pradeau, excellent traducteur et exégète de Platon, ce livre donne un aperçu très nuancé des débats actuels.

Le terme lui-même, "formes intelligibles », est à plusieurs égards une nouveauté. Personne ne parle plus d'idées, même si le lexique de Platon, qui demeure très complexe, l'autoriserait pleinement. Profondément marquée par la lecture néokantienne d'un Paul Natorp, pour ne citer que lui, l'interprétation du tournant du vingtième siècle avait pris le risque de faire des "formes" des contenus mentaux, susceptibles, selon certains, de renouveler la lecture de l'épistémologie kantienne. Pour les interprètes de l'école de Marbourg, en effet, les formes devenaient des concepts, c'est-à-dire des structures logiques. Il revient à Harold Cherniss, sans doute le platonisant le plus orthodoxe et le plus rigoureux du vingtième siècle, d'avoir montré une fois pour toutes que les formes possèdent une extériorité ontologique qui leur est assurée par leur statut d'objets séparés. Tous les prédicats par lesquels Platon les introduit et les analyse, y compris ceux qui proviennent de la mythologie, comme la divinité ou l'immortalité, convergent dans cette direction, et l'interprétation mentaliste, ou idéaliste, introduite déjà dans le moyen platonisme d'un Albinus et poursuivie dans le néo-kantisme, a fait son temps. Ce qui ne signifie pas que le problème de la substantialité des formes soit pour autant résolu.

Ce livre nous donne la traduction d'une importante étude de Cherniss, publiée pour la première fois en 1936: L'économie philosophique de la théorie des idées. Le traducteur a conservé ici le mot de Cherniss en anglais, ideas, mais l'interprétation n'a rien à voir avec quelque mentalisme que ce soit. Cherniss insiste en effet sur la fonction des formes intelligibles, en ce qu'elles offrent à la pensée le fondement ontologique stable, nécessaire à l'épistémologie réaliste. Cherniss pense en effet que l'hypothèse des formes intelligibles est d'abord formulée pour résoudre, de manière unitaire, des problèmes éthiques, épistémologiques et ontologiques, qui, chacun dans leur sphère, présentaient les mêmes apories. Son analyse isole donc ce qui, dans les formes, permet de garantir un fondement dans chacun de ces trois domaines: les valeurs, la connaissance, l'être. Seule cette hypothèse permet en effet de concevoir ces ordres de l'existence comme aspects d'un unique cosmos unifié. Ce texte demeure un classique, et il faut espérer que cette traduction très claire contribuera à le diffuser auprès de ceux qui ont pour tâche de faire connaître Platon à des débutants. Tous ceux en effet qui désirent faire leur chemin dans l'interprétation de cette doctrine sont pour ainsi dire mis en position d'avoir à se placer par rapport à l'interprétation standard proposée par Cherniss. 
Le travail de Harold Cherniss, à qui nous devons l'imposante Bibliographie de Platon ${ }^{1}$ poursuivie depuis par Luc Brisson, ne s'est pas élaboré dans les milieux qui, au vingtième siècle, ont vu naître les deux grands paradigmes de l'interprétation platonicienne, et il ne leur doit rien; d'une part, son renouvellement dans l'analyse logico-sémantique, qui fut le résultat de la philosophie analytique, et d'autre part le débat, assurément passionné, qui, dans la foulée des recherches de l'École dite de Tübingen, menées par Hans-Joachim Krämer et Konrad Gaiser, a associé l'interprétation de la doctrine des formes aux énigmes de la doctrine du Bien et de l'Un. Dans le présent recueil, on trouvera d'excellentes discussions des problèmes qui sont communs à ces débats, mais on peut regretter qu'aucun chapitre ne soit consacré à exposer et discuter ces deux ensembles pour eux-mêmes. En ce qui concerne l'approche logico-analytique, il faut y reconnaître, comme le signale à juste titre Jean-François Pradeau, un indice de la difficulté, incontournable dans le texte de Platon, de trouver une affirmation ontologique sans ambiguité de la nature des formes. Le questionnement sur leur réalité est donc souvent ramené, dans la pensée analytique, à une interprétation qui en fait des "paradigmes sémantiques, des prédicats qu'on attribue aux réalités sensibles ». Cette interprétation heurte de front la majorité des textes de Platon, mais elle profite de certaines failles dans la construction des prédicats, surtout dans les premiers dialogues, pour soutenir que Platon a durci une intuition analytique très productive, en l'assortissant d'une métaphysique des genres tout à fait insoutenable. Comme Pradeau le remarque, cette interprétation a connu une grande diffusion; elle est actuellement dominante, et il ne faut pas s'en étonner: elle produit en effet une lecture de Platon dans laquelle la métaphysique d'un monde intelligible séparé, incluant la question de l'âme, est devenue marginale. Tout se passe en effet comme si on répondait à la question: à quoi pourrait nous servir cette doctrine aujourd'hui ?, au lieu de se demander comment elle s'était constituée pour Platon. Quant à l'approche de Tübingen, on ne peut pas dire qu'elle s'attache directement à l'ontologie des formes, mais plutôt à la doctrine des genres. Dans la mesure où le présent recueil paraît avoir voulu se concentrer sur le problème de la participation, on peut comprendre que la question des genres ait été laissée un peu à l'écart.

Jean-François Pradeau ouvre le recueil avec une étude minutieuse du lexique de l'eidos. Son analyse parcourt l'ensemble du corpus pour en retracer les occurrences, pour tenter de déterminer la signification du terme selon les usages et le mettre en rapport avec la question de la participation. La forme, en effet, est un être intelligible auquel le monde sensible participe d'une certaine manière. La lecture proposée ici veut conduire à une distinction qui permettrait de reconnaître dans les formes intelligibles des déterminations particulières et de les considérer comme distinctes de la réalité intelligible en tant que telle. Selon Pradeau, «la forme est la détermination qui est donnée à une multiplicité de choses sensibles par

1. Harold Cherniss, Plato 1950-1957, Lustrum 4 \& 5 (1959 \& 1960); Luc Brisson, Platon 1958-1975, Lustrum 20 (1977); Luc Brisson, en collaboration avec Hélène Ioannidi, Platon 1975-1980, Lustrum 25 (1983) 31-320, avec des Corrigenda à Platon 1975-1980, Lustrum 26 (1984) 205-206; Platon 1980-1985, Lustrum 30 (1988) 11-294, avec des Corrigenda à Platon 1980-1985, Lustrum 31 (1989) 270-271; Platon 1985-1990, Lustrum 35 (1993), et Luc Brisson, avec la collaboration de Frédéric Plin. Platon 1990-1995. Paris, Librairie Philosophique J. Vrin, 1999, avec des Addenda aux tranches antérieures, 407-415. 
la réalité intelligible qui est une qualité singulière». Cette recherche se situe donc au croisement de l'analyse logico-sémantique et de la métaphysique, et c'est ce qui lui confère son originalité. Pradeau analyse en effet méticuleusement le texte crucial du Ménon (72a6-73c10), dans lequel il fait ressortir cette distinction entre la détermination de l'eidos comme qualité, saisie notamment dans l'appartenance d'individus à une même espèce, et l'eidos comme cause, c'est-à-dire comme réalité intelligible. La possibilité d'une distinction entre les réalités intelligibles et les formes intelligibles devient alors l'objet d'une intéressante discussion; Pradeau a raison de critiquer la position de ceux qui ne voient aucune différence entre le Beau en soi et la Forme du Beau, et les conséquences pour l'interprétation de la participation sont décisives. C'est en effet d'abord la forme (eidos) qui est participée, et non la réalité intelligible en tant que telle, c'est-à-dire l'ousia. Ce chapitre emprunte beaucoup à la problématique analytique des particularités, et on peut y reconnaître un effort d'une grande rigueur pour parvenir à une interprétation synthétique. Quant à moi, je ne suis pas certain de ne pas y reconnaître certains biais néoplatoniciens, notamment dans ce qui pourrait subsumer un intellect, mais nul ne peut contester la réalité de la distinction introduite dans cette analyse.

Il revient à Luc Brisson, interprète et traducteur éminent de Platon, d'avoir pris le relais du platonisme standard de Harold Cherniss et de continuer de défendre, contre des interprétations purement logiques, une lecture accordée avec l'ensemble de la métaphysique du monde intelligible. Sa contribution au présent recueil est une discussion très fine de la question de la participation du sensible à l'intelligible. Après avoir posé le problème dans le texte de Platon, et notamment à partir de l'exposé du Timée et du Phédon, où la participation est présentée comme causalité exemplaire et interprétée comme présence, Brisson introduit le grand texte critique du Parménide qui met en relief les apories de la présence mais retient la causalité sous une modalité de ressemblance. Cette première interrogation sur la participation conduit cependant à une position aporétique puisqu'elle ne peut expliquer la multiplicité et la diversité du sensible; c'est seulement dans l'hypothèse du Démiurge du Timée que ces questions recevront une réponse, même si celle-ci est affectée d'un quotient de vraisemblance tributaire du récit. Mais comme l'exposé très complet de Brisson le montre, là où le Parménide permet de comprendre la relation entre l'image et le modèle, seul le Timée permet d'accéder au questionnement sur la genèse du monde sensible et la constitution de l'univers. En peu de pages, Luc Brisson montre ici une maîtrise exceptionnelle de la théorie, et sa contribution propose un équilibre très rigoureux entre deux lectures, toujours compatibles, dans la dernière période de l'œuvre platonicienne.

L'exposé de Walter G. Leszl, un interprète brillant, connu pour ses riches travaux sur Aristote, se concentre sur les divers modèles d'interprétation de la doctrine métaphysique des formes dans les dialogues de maturité. L'auteur intervient avec beaucoup de justesse, comme il est maintenant de rigueur de le faire, sur le contexte pragmatique de l'émergence de la doctrine: la réfutation du relativisme et du scepticisme. L'exposé perd cependant un peu de son tranchant, l'auteur ayant pris le parti d'exposer la doctrine de la nouvelle Académie pour comprendre à rebours le scepticisme de Platon lui-même. L'analyse des passages sur l'illusion et la perspective présente plusieurs aperçus très riches qui permettent de renouveler les contextes et de préciser la doctrine des apparences. Ce chapitre s'éloigne sans doute un peu des objectifs propédeutiques de la collection, et il ne sera pas facilement 
accessible aux débutants. Ses conclusions sont cependant claires: la formulation même de la doctrine de l'eidos doit beaucoup, sinon tout, aux apories de l'illusion et de la perspective, dans la mesure où on ne peut qu'y reconnaître une doctrine de la figure. De belles pages sur l'importance de la doctrine de l'âme auraient mérité un traitement autonome.

Il revenait enfin à Francesco Fronterotta d'exposer la critique aristotélicienne. Dans un livre qui se termine par un texte de Cherniss, dont l'ouvrage central est l'exposé de cette question, le défi était considérable, et cet article le relève de manière lumineuse. L'auteur présente avec précision le corpus des textes d'Aristote où cette critique est formulée, et il en propose une interprétation systématique, en en reprenant de manière formelle les arguments. Cette partie de son article est remarquable de clarté, et le recours au dialogue de jeunesse d'Aristote, De Ideis, tout à fait pertinent. Comme l'auteur le dit avec justesse, la critique aristotélicienne était impitoyable, et elle a conduit Aristote à sa propre conception de l'eidos dans les livres Z-H de la Métaphysique. C'est dans l'exposé de sa conception de la forme qu'on trouve en fait la vraie réponse à Platon.

Ce recueil apporte à la discussion contemporaine un instrument de travail de premier calibre; il est complété de deux courts index, et on peut regretter l'absence de bibliographie (légèrement compensée par des notes souvent très substantielles à cet égard). L'érudition sur ces questions, comme pour tant d'autres, est devenue immaîtrisable, et ce livre fournit un guide sûr pour y faire son chemin.

GEORGES LEROUX

Université du Québec à Montréal

\section{Bernard Baertschi et Kevin Mulligan (dir.), Les nationalismes, Paris, Presses Universitaires de France, collection Éthique et philosophie morale, 2002, 251 pages.}

Cet ouvrage propose quelques pistes pour remédier aux conflits ethniques et nationaux tout en rejetant la solution, jugée inadéquate, qui consiste à éliminer les particularismes de l'espace politique public. Il s'agit d'un recueil rassemblant des textes écrits durant la décennie précédente. L'équilibre recherché est celui d'un "amour du pays» qui ne reconduit pas les tares du "préjugé national ", pour reprendre un mot d'Adam Smith (p. 4).

Le recueil est divisé en trois parties. La première, intitulée Les forces $d u$ nationalisme, contient les textes de Philip Gerans, qui compare diverses formes historiques de nationalisme; de David Miller, qui défend la "nationalité » et de Bernard Baertschi, sur "Le charme secret du patriotisme". La seconde, Les faiblesses $d u$ nationalisme, contient ceux de Daniel M. Weinstock, sur une défense morale du nationalisme; de Ninad Miscevic, sur les difficultés à justifier le nationalisme et d'Elmar Holeinstein sur le concept de Kulturnation. Enfin, la dernière, Quelques remèdes, regroupe les textes de Markus Haller sur le multiculturalisme, de Barry Smith, qui analyse la nation à partir de sa "spatialité » et enfin, de Murray N. Rothbard, sur une conception libertarienne de la nation.

Cette élégante tripartition vise à déployer à la fois les forces et les faiblesses $\mathrm{du}$ nationalisme en proposant, finalement, des solutions qui s'inspirent d'une 